\title{
Design and Learning Effectiveness Evaluation of Gamification in e-Learning Systems
}

\author{
Mohammad T. Alshammari \\ College of Computer Science and Engineering \\ University of Hail, Hail, Saudi Arabia
}

\begin{abstract}
This paper proposes a gamification design model that can be used to design and develop gamified e-learning systems. Furthermore, a controlled and carefully designed experimental evaluation in terms of learning effectiveness of gamification is offered. The experiment was conducted with 44 participants randomly assigned to an experimental 'gamified' condition and a controlled 'non-gamified' condition. In both conditions the same learning material, to teach computer security, were used. The main difference between the two conditions was the integration of gamification in an e-learning system designed based on the proposed model. The results indicate that learning using the gamified version of the e-learning system produces better short-term and medium-term learning gain than learning using the non-gamified e-learning version. Future avenues of research are also provided.
\end{abstract}

Keywords-Gamification; e-learning systems; interaction design; experimental evaluation

\section{INTRODUCTION}

Learning is a complex issue that can be influenced by different factors including learner characteristics, learning content, learning environment and teaching style. Traditional learning approaches through classrooms can be rigid and unattractive to some learners given the time and place constraints. In contrast, e-learning systems provide the ability for learners to learn anytime, anywhere and offer different interactivity levels that may not well be supported by traditional learning approaches. Moreover, learning content can be incorporated in e-learning systems with different types such as examples, simulations, problem-solving tasks and explanations. These types can also be offered in different multimedia formats including written and spoken material in addition to videos and games.

Motivating learners to use such systems in order to enhance their learning and gain some knowledge is a challenging task [1]. Gamification is usually put forward as a proposed solution to motivate, engage and support learners through their interaction with learning material [2]. The term 'gamification' was firstly introduced in the scientific community in 2010 [3]. Since then, it is still widely adopted by researchers in different research domains and areas including learning, business and health [4]. Gamification can be defined as the use of game elements, thinking and mechanisms in a non-game context [3].

E-learning systems can be designed to integrate different game elements such as points, badges, progress bars and levels to support learners in having a gamified learning experience [5]. Gamification in learning should be differentiated form the concept of game-based learning which principally aims at delivering learning content and objectives in an entertaining approach implemented as a game so that learners can play the game in order to learn.

Based on the literature and recent reviews on gamification [4], [14], [15], several issues have been found. First, most gamification studies were not supported with empirical evidence following well-designed, controlled and thorough experimental evaluations that have control conditions. Second, mixed results can also be found generally with limited sample sizes. Third, studies were mostly based on short-term applications of some types of game elements. Fourth, some studies do not rely on such models as a foundation to the design and development of gamified e-learning systems. This issue can be challenging when other researchers need to replicate their work. Furthermore, gamification is still in its infancy and quickly developing requiring more focused and thorough studies [14].

These issues need to be carefully addressed when applying gamification in learning. This paper bridges this gap by offering a gamification design model that can be used to develop different instances of gamified e-learning systems. The model is consisted of three major components including the courseware module, the learner profile and the gamification component. The paper also offers an evaluation of gamification in an e-learning system, developed based on the proposed model. A carefully designed and controlled experimental evaluation was conducted with 44 participants in a real learning environment. The participants used the developed gamified e-learning system to learn some concepts related to a course on computer security, the application domain. This study primarily aims to explore the learning effectiveness of the integration of gamification in e-learning systems. The main research question of this study is that does the integration of gamification in e-learning systems enhance learning?

The rest of the paper is structured as follows. Section II provides related work to gamification in e-learning systems. Section III details the proposed gamification design model. Section IV outlines the evaluation method. Section V offers the results. Section VI concludes the paper highlighting the main findings. Section VII points out to future directions of research.

\section{RELATED WORK}

Many attempts have been proposed to investigate gamification in online-learning research. For instance, a blended study (classroom and online) conducted by [6] to evaluate the learning effect of gamification yielding positive 
results. In [7], design phases were proposed to integrate gamification in e-learning systems supported with some results related mainly to learning outcomes. Gamified quizzes besides lectures were also offered in [8]; there were positive results in terms of perceived usefulness of gamification based on a survey. Yet, quizzes can represent one aspect of learning so that they have not focused on the whole learning process. A gamified e-learning system to teach Java programming language was also developed and evaluated in terms of learning gain and motivation [9]. However, their results cannot be generalized since their study was applied only to first year undergraduate students.

Moreover, research in [10] compared different forms of gamification including competitive, collaborative and adaptive gamified learning activities concluding that gamification can be beneficial in learning when diverse game elements are incorporated in e-learning systems. However, their study used four experimental conditions where each condition was lasted for a very short learning time affecting the controllability and feasibility of their experiment. Research in [11] investigated gamification based on feedback as a single game element yielding negative results; they argued that the integration and arrangements of various game elements can be more valuable in learning. Similarly, the work in [12] investigated the effect of badges as the key game element on motivation and performance of learners without yielding positive findings. The effort made by [13] focused on gamifying learning activities to learn some concepts of $\mathrm{C}$-programming language producing encouraging results in terms of learner achievements and engagement. However, the work of [13] was based on a very small size so that their results cannot be generalized and further studies with more participants are needed. Accordingly, more careful studies are needed to investigate the effect of gamification in e-learning systems which justify the work presented in this paper.

\section{GAMIFICATION DESIGN MODEL}

It is important to highlight how gamification can be designed in e-learning systems before attempting to investigate their effect on learning [3], [7]. Therefore, a gamification design model is proposed and depicted in Fig. 1. The model is consisted of a number of components. It mainly includes the gamification component, the courseware module and the learner profile. Information stored in these components can be retrieved by the gamification business logic in order to generate the gamified learning experience, and then deliver it to the learner. The output also feeds into the learner profile for updates.

In the proposed model, there are also three stakeholders: learner, instructor and admin. The learner is the person who is conducting some tasks in order to gain some knowledge and understanding of something. The instructor is the person who facilitates the creation of such courses (objectives, units, lessons and assessments) to support learners. The admin can manage the implemented model by handling the technological perspectives, and by adding gamification elements. The admin can also be enabled to play the role of an instructor in the model.

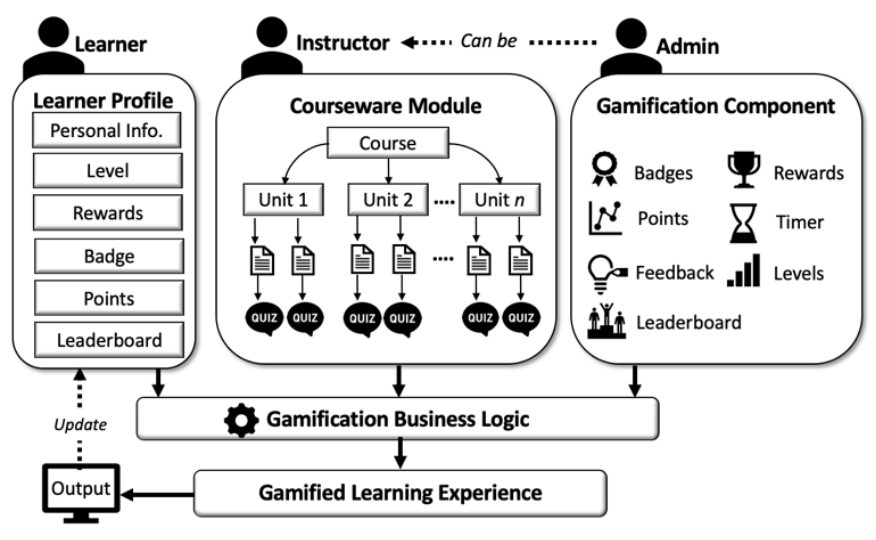

Fig. 1. The Gamification Design Model.

\section{A. Gamification Component}

The gamification component aims to represent, manage and maintain the gamification elements by system admins. There are seven main game elements in the gamification component including badges, rewards, points, timer, feedback, levels and leaderboard because they are widely used in related work [1]. Each element is described and presented in Table I.

The model, when implemented, can be flexible in a way that enables admins to create and maintain different information related to each element easily. For example, the admin can create a number of levels, name each level and then store them in the system such as beginner level, intermediate level and advanced level. Another example, badges can be created to unlimited number assigning a picture and a title to each one. Different rules and requirements can be applied to all the elements in the gamification component.

It is important to note that these game elements can be created and maintained in isolation from other components of the model. Though, they can easily be assigned to each learning activity. This is to allow instructors to mainly focus on the creation of courses and learning activities at the first place. Then, they can map the game elements to learning activities by selecting suitable ones as preferred. Moreover, not all elements are necessarily be activated in the system but rather enabling instructors to select elements that are expected to enhance learning and motivation and learners.

TABLE. I. DESCRIPTION OF GAMIFE ELEMENTS

\begin{tabular}{|l|l|}
\hline Game Element & Description \\
\hline Badges & $\begin{array}{l}\text { A distinctive emblem awarded when accomplishing } \\
\text { specified learning tasks. }\end{array}$ \\
\hline Rewards & $\begin{array}{l}\text { A stimulus given based upon successful completion of a } \\
\text { particular learning task. }\end{array}$ \\
\hline Points & $\begin{array}{l}\text { Points are incremented based on the learner progress } \\
\text { toward learning. }\end{array}$ \\
\hline Feedback & $\begin{array}{l}\text { A set of timers created to be mapped to learning lessons } \\
\text { and tests. }\end{array}$ \\
\hline Levels & $\begin{array}{l}\text { The provision of corrective information for learning } \\
\text { misconception based on the results of tests. }\end{array}$ \\
\hline Leaderboard & $\begin{array}{l}\text { A position of the learner on a pre-defined scale based } \\
\text { upon the learning progress. }\end{array}$ \\
\hline
\end{tabular}




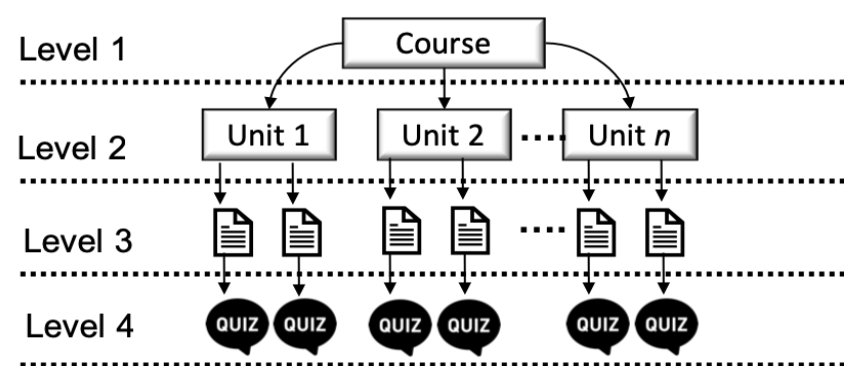

Fig. 2. The Courseware Module Structure with Four Levels. Level 1 Contains the Course Node. Level 2 has a Number of Learning units. Level 3 Involves Some Learning Lessons Associated with Related Learning units.

Level 4 is consisted of a Number of Quizzes, and Each Quiz can be Linked to a Particular Learning Lesson.

\section{B. Courseware Module}

The courseware module is an important component in the gamification design model. Its main purpose is to represent, store and maintain learning material related to a specific course. It uses a hierarchical representation in a tree-like structure with four levels as shown in Fig. 2.

The root or level 1 represents the course node. It contains information or metadata related to the course such as the title, description, keywords and objectives to describe the course. Level 2, which entirely connected to the course node, is consisted of a number of learning units. Each unit node has some metadata to define its properties the same as the course node. A number of learning lessons can be linked to specific learning units. These lessons are located in level 3 and have additional metadata such as pre-requisites, type and multimedia used. Quizzes can also be created and then explicitly be mapped to specific learning lessons in level 4.

It is important to highlight the point that the hierarchical structure representation of the courseware module can enable instructors to author learning content in a more organized way without thinking of how to integrate gamification through the learning content. This particular representation is helpful as learning activities can automatically or manually be mapped to game elements. In addition, it is possible to generate distinctive courses related to different learning areas supporting the generalization of the proposed model and its components.

\section{Learner Profile}

According to [16], a learner profile is "what enables a system to care about a student". The learner profile represents learner characteristics and maintains the learner level, badges, rewards, leaderboard and points. The information stored in the learner profile is continually updated as the learner progresses through learning using the gamified e-learning system. For example, when a learner successfully studies a lesson and completes a quiz related to that lesson, some points can be added to the learner profile depending on the quiz score and time spent. Accordingly, when a set of quizzes are completed by a learner, other gamification elements can be updated and then stored in the learner profile.

\section{METHOD}

This section details the methodology used to evaluate the effectiveness of gamification on learning. A gamified e- learning system was developed taken into account the proposed gamification design model to answer the main research question. Fig. 3 shows a screenshot example of the gamified system. This example shows the profile of the learner highlighting the rank of the learner among his/her peers, the number of awards obtained, the number of points earned, the medal achieved which is a silver medal and the level of the student (i.e., a beginner level). The main purpose of developing the system is to validate the proposed model, and to evaluate the learning effectiveness of gamification through a controlled experiment. All the seven game elements in the model were incorporated in the system to offer a complete gamified learning experience to learners.

A pilot testing was conducted in two sessions prior to the main controlled experiment with 10 participants (5 students, 3 instructors and 2 interaction design experts) to learn some topics related to computer security (i.e., the application domain of the system) and to walkthrough the implemented system. This is to mimic the actual experiment, to find out issues that may occur and to ensure that the system is working properly. All participants in the pilot testing contributed and commented on some ways to improve the system, the measurement tools and learning material. By the pilot testing, it was also possible to indicate the time needed to complete the experiment. Some amendments were made before conducting the main experiment according to the findings and observations of the pilot test.

\section{A. Hypotheses}

Two main hypotheses are proposed for this study as follows:

- Hypothesis (H1). Learning using the gamified version of the e-learning system produces better (short-term) learning gain than learning using the non-gamified version.

- Hypothesis (H2). Learning using the gamified version of the e-learning system produces better (medium-term) learning gain than learning using the non-gamified version.

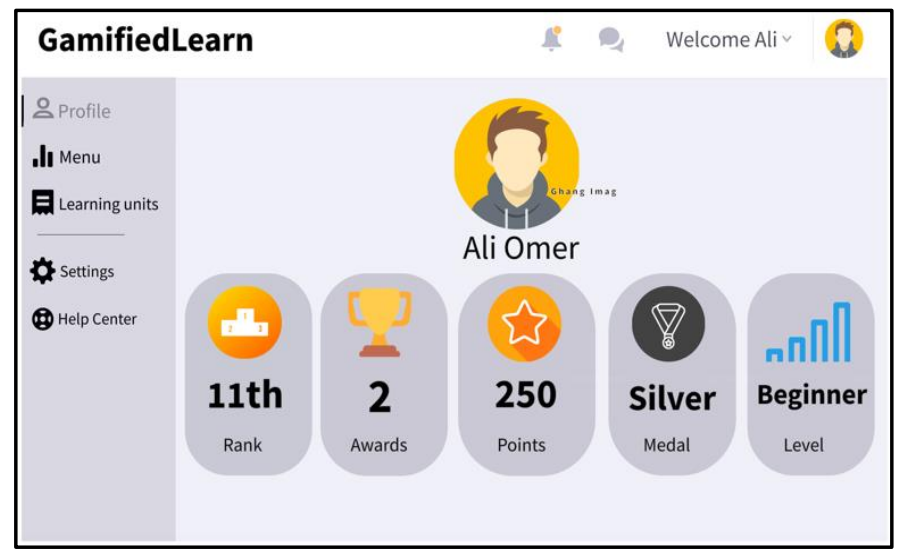

Fig. 3. A Screenshot Example of the Gamified e-Learning System. this Example Shows the Profile of The Learner Highlighting the Rank of the Learner Among his/her Peers, the Number of Awards Obtained, the Number of Points Earned, the Medal Achieved which is a Silver Medal and the Level of the Student (Beginner). 


\section{B. Measurement Tools}

Learning gain was evaluated using three main tests: pretest, post-test and follow-up test. Two types of learning gain are measured based on the tests. First, short-term learning gain is measured as the difference between the post-test and the pretest in order to evaluate the immediate learning effectiveness of gamification. Second, medium-term learning gain is calculated as the difference between the follow-up test and the pre-test in order to evaluate the sustained knowledge. Each test involves 25 items related to a course on computer security. Each item has five multiple-choices with the fifth being 'I don't know!' in order to reduce the chance of random speculating by participants.

It should be noted that all tests are similar except for the ordering, arrangements and phrasing of both the items and the responses. A Cronbach alpha was also measured for the pretest, post-test and follow-up test having good reliability as 0.96 , 0.82 and 0.83 , respectively.

\section{Experimental Procedure}

Participants were first welcomed and introduced to the process of the experiment after signing consent forms of participation. Then, all participants were randomly assigned to one of two conditions: (A) a gamified version of the e-learning system (experimental) or (B) a non-gamified version (control). Participants in both A and B completed the pre-test, and then studied some learning material related to computer security using the system according to their assigned condition. The learning material in both conditions are similar and the difference is whether students were exposed to gamification or not based on their assigned condition.

The learning task was completed in a computer laboratory for three weeks where there were two experimental sessions per week. Each session was lasted about 120-180 minutes. At the end of all the experimental sessions, participants were immediately directed to complete the post-test. Two to three weeks after completing the main experiments, all participants were invited to only complete the follow-up test.

\section{Data Analysis}

The data of the experiments were collected by the system automatically and stored in a database. Then, they were converted into a format that enables for the data analysis process. Minitab 19 software was used for data analysis. The type of the tests performed is outlined when presenting the results in the following section.

\section{RESUlTS}

A number of 44 Male undergraduate students form the College of Computer Science and Engineering, University of Hail, Saudi Arabia completed the experiments. The gender variable was controlled to eliminate any variance and confounding effect on the results. The conditions being experimental 'gamified' and control 'non-gamified' were balanced having 22 participants each.

There were five variables related to learning gain (pre-test, post-test, follow-up test, short-term learning gain and mediumterm learning gain) in the experiment. Fig. 4 summarizes the mean score results for those variables.

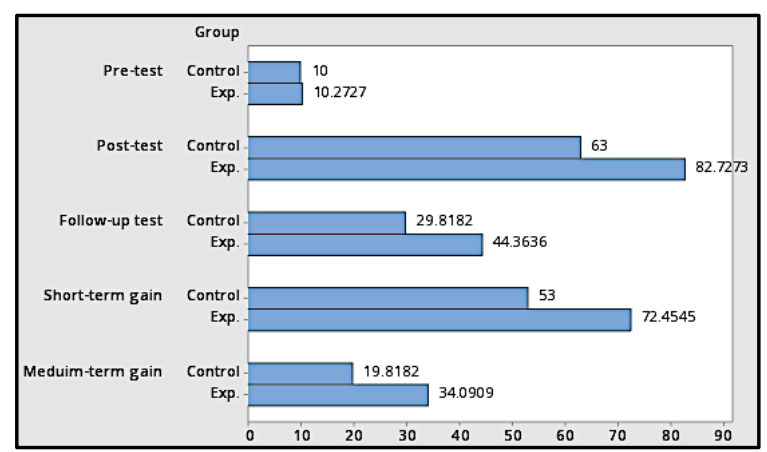

Fig. 4. Summary for the Mean Scores of the Experimental Variables.

Regarding the pre-test results, an independent sample t-test was conducted to compare the group who interacted with the gamified version of the e-learning system and the non-gamified version. There was no statistically significant difference in the scores for the gamified group $(\mathrm{M}=10.27, \mathrm{SD}=3.92)$ and the non-gamified group $(\mathrm{M}=10, \mathrm{SD}=4.58) ; \mathrm{t}(41)=-0.21, \mathrm{p}=0.83$. These results suggest that all participants had the same knowledge level prior to the experiment which enabled for more reasonable comparison between the two experimental conditions. Hence, any positive learning effect found was primarily caused by using the gamified e-learning system.

Reporting on the post-test results, there was a statistically significant difference in the scores for the gamified group $(M=82.73, S D=9.83)$ and the non-gamified group $(M=63$, $\mathrm{SD}=14.3) ; \mathrm{t}(37)=-5.32, \mathrm{p}=0.000$, according to an independent sample t-test. These results suggest that participants who used the gamified e-learning system achieved better learning gain than those in the non-gamified version.

An independent sample t-test was also conducted concerning the follow-up test. The results indicated a statistically significant difference in the scores for the gamified group $(\mathrm{M}=44.4, \mathrm{SD}=10.4)$ and the non-gamified group $(\mathrm{M}=29.8, \mathrm{SD}=15.2) ; \mathrm{t}(37)=-3.71, \mathrm{p}=0.001$. The learning gain scores according to the follow-up test in comparison to the post-test scores have been decreased by about $50 \%$ in both conditions because participants took the follow-up test after the experiment two to three weeks later affecting the sustained knowledge. Still, the results suggested positive findings related to the experimental 'gamified' condition.

By obtaining the results related to the pre-test, post-test and follow-up test, it is possible to test the hypotheses $\mathrm{H} 1$ and $\mathrm{H} 2$. Regarding the short-term learning gain, calculated as the difference between the post-test scores and the pre-test scores, an independent sample t-test was conducted. There was a statistically significant difference in the short-term learning gain scores for the gamified group $(\mathrm{M}=72.5, \mathrm{SD}=10.5)$ and the non-gamified group $(\mathrm{M}=53, \mathrm{SD}=14.7) ; \mathrm{t}(37)=-5.04, \mathrm{p}=0.000$. According to the results, the hypothesis $\mathrm{H} 1$ can be confirmed. It can be suggested that learning using the gamified version of the e-learning system produces better (short-term) learning gain than learning using the non-gamified version.

Reporting on the medium-term learning gain based on the conducted independent sample t-test, there was also a statistically significant difference in the medium-term learning gain scores for the gamified group $(\mathrm{M}=34.1, \mathrm{SD}=11.8)$ and the 
non-gamified group $(\mathrm{M}=19.8, \mathrm{SD}=15.9)$; $\mathrm{t}(37)=-3.39, \mathrm{p}=0.002$. Based on the results, the hypothesis $\mathrm{H} 2$ can be confirmed. It is suggested that learning using the gamified version of the elearning system produces better (medium-term) learning gain than learning using the non-gamified version.

\section{CONCLUSION}

This paper contributed to the current literature by the provision of the gamification design model. The model can be used as a foundation to design different instances of gamified e-learning systems. It is consisted of major components needed to develop such systems including the learner profile, the courseware module and the gamification component. An implementation of the model was also completed resulting into a gamified e-learning system in order to validate the proposed model and to evaluate the learning effectiveness of gamification.

Moreover, a thorough experimental evaluation in terms of learning gain was conducted considering the limitations of published research. Learning gain was also reported not only according to the immediate (short-term) learning effect measured by a post-test taken by participants after completing the experiment, but also according to a delayed (medium-term) learning effect measured based on a follow-up test taken by participants two to three weeks later after completing the experiment. The findings indicated that learning using the gamified version of the e-learning system produces better (short-term and medium-term) learning gain than learning using the non-gamified e-learning version.

\section{VII.FUTURE WORK}

Pointing out to future work, it is planned to conduct a longer-term evaluation in a semester long duration adding more learning resources and focusing on another application domain in order to generalize the current findings with a larger sample size. It is also true that this paper reports on learning gain as an important variable; yet, other variables can be considered in future experiments such as motivation and satisfaction since the study presented in this paper builds up the foundation to conduct more experiments by the proposed model and by the careful experimental evaluation approach.

Another potential future direction is to compare the effect of unique combination of different game elements on different cognition and psychological factors with careful considerations to experimental design. Another possible research direction is to investigate different personalities and learning style of learners and to explore how they relate to certain game elements. Also, the culture and the learning context can play an important role in motivating or demotivating learners to use gamified e-learning systems. Therefore, more research is required to compare the effect of using gamified e-learning systems in different learning contexts and cultures. Additionally, researchers can conduct more research to explore the extent to which learners are engaged in the learning process when using gamified systems in long-term evaluation studies. Moreover, the proposed gamification model can further be improved to cater for collaborative and social learning besides the integration of game elements in e-learning systems to support active learning. An essential point to emphasize, generally in online-learning research, is that gamified elearning systems should be designed following sound instructional theories and models.

\section{REFERENCES}

[1] M. Sailer, J. U. Hense, S. K. Mayr, and H. Mandl, "How gamification motivates: An experimental study of the effects of specific game design elements on psychological need satisfaction," Computers in Human Behavior, vol. 69, pp. 371-380, 2017.

[2] I. V Osipov, E. Nikulchev, A. A. Volinsky, and A. Y. Prasikova, "Study of gamification effectiveness in online e-learning systems," International Journal of advanced computer science and applications, vol. 6, no. 2, pp. 71-77, 2015.

[3] S. Deterding, D. Dixon, R. Khaled, and L. Nacke, "From game design elements to gamefulness: defining gamification," in Proceedings of the 15th international academic MindTrek conference: Envisioning future media environments, 2011, pp. 9-15.

[4] G. Baptista and T. Oliveira, "Gamification and serious games: A literature meta-analysis and integrative model," Computers in Human Behavior, vol. 92, pp. 306-315, 2019.

[5] I. Varannai, P. L. Sasvári, and A. Urbanovics, "The use of gamification in higher education: an empirical study," International Journal of Advanced Computer Science and Applications, vol. 8, no. 10, pp. 1-6, 2017.

[6] M. Tan and K. F. Hew, "Incorporating meaningful gamification in a blended learning research methods class: Examining student learning, engagement, and affective outcomes," Australasian Journal of Educational Technology, vol. 32, no. 5, 2016.

[7] D. Strmečki, A. Bernik, and D. Radošević, "Gamification in e-Learning: introducing gamified design elements into e-learning systems," Journal of Computer Science, vol. 11, no. 12, pp. 1108-1117, 2015.

[8] J. Filippou, C. Cheong, and F. Cheong, "A Model to Investigate Preference for Use of Gamification in a Learning Activity," Australasian Journal of Information Systems, vol. 22, 2018.

[9] F. L. Khaleel, N. S. Ashaari, and T. S. M. T. Wook, "An empirical study on gamification for learning programming language website," Jurnal Teknologi, vol. 81, no. 2, 2019.

[10] T. Jagušt, I. Botički, and H.-J. So, "Examining competitive, collaborative and adaptive gamification in young learners' math learning," Computers \& Education, vol. 125, pp. 444-457, 2018.

[11] K. Welbers, E. A. Konijn, C. Burgers, A. B. de Vaate, A. Eden, and B. C. Brugman, "Gamification as a tool for engaging student learning: A field experiment with a gamified app," E-Learning and Digital Media, vol. 16, no. 2, pp. 92-109, 2019.

[12] E. Kyewski and N. C. Krämer, "To gamify or not to gamify? An experimental field study of the influence of badges on motivation, activity, and performance in an online learning course," Computers \& Education, vol. 118, pp. 25-37, 2018.

[13] M.-B. Ibanez, A. Di-Serio, and C. Delgado-Kloos, "Gamification for engaging computer science students in learning activities: A case study," IEEE Transactions on learning technologies, vol. 7, no. 3, pp. 291-301, 2014.

[14] J. Koivisto and J. Hamari, "The rise of motivational information systems: A review of gamification research," International Journal of Information Management, vol. 45, pp. 191-210, 2019.

[15] S. Subhash and E. A. Cudney, "Gamified learning in higher education: A systematic review of the literature," Computers in Human Behavior, vol. 87, pp. 192-206, 2018.

[16] J. A. Self, "The defining characteristics of intelligent tutoring systems research: ITSs care, precisely," International Journal of Artificial Intelligence in Education, vol. 10, pp. 350-364, 1999. 